\title{
Логический анализ дефинициальной дедукции ${ }^{1}$
}

\author{
В. И. ШАЛАК
}

\begin{abstract}
The article is devoted to analisis of definitions and their properties in type-free languages. In order to achieve this aim we define logic of definitional deduction. The single rule of this logic is replacement of terms according to their definitions. The resulting logic is definitionally equivalent to combinatory logic of Schönfinkel-Curry.
\end{abstract}

Ключевые слова: определение, дедукция, комбинаторная логика, неподвижная точка.

\section{1 Введение}

Если историю возникновения логики вести со времен Платона и Аристотеля, то окажется, что одновременно это была и история теории определений, которая традиционно считается одним из разделов логики. Цель настоящей работы заключается в том, чтобы еще раз обратить внимание на роль определений в процессе познания и проанализировать возможности их использования в логических рассуждениях.

Достаточно распространено убеждение, что теория определений имеет вполне завершенный вид и ничего принципиально нового дать нам не может, что основная ее задача - классифицировать определения по видам, уточняя условия правильности их применения. Как иронически замечает Дж. Браун, "Чтобъ вызвать зевоту у читателя, трудно найти более подходящую тему, чем определения" [1].

Наиболее распространенными являются явные определения, которые имеют простую лингвистическую форму,

$$
A={ }_{\text {def }} B .
$$

${ }^{1}$ Работа выполнена при поддержке РГНФ, грант № 08-03-00173а 
Левая часть этого выражения, обозначенная буквой А, называется определяемым (дефиниендумом), а правая часть, обозначенная буквой $\mathrm{B},-$ определяющим (дефиниенсом). Символ

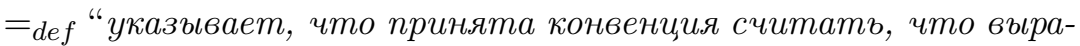
жение A означает то же самое, что и выражение B" [2]. В левой части определения обязательно присутствует новый так называемый определяемый термин, который вводится в язык, расширяя его.

Очень часто, если говорить о формальной стороне, явные определения рассматривают всего лишь как некоторые сокращения других комбинаций символов.

"Определение - это декларация о том, что вновь введенный символ или комбинация символов означает то же самое, что и некоторая другая комбинация символов, значение которой уже известно" [3].

Поэтому они не могут привнести с собой ничего нового.

"После того, как посредством определения некоторый знак получил значение, он его отныне имеет, и определение переходит в предложение, в котором утверждается тождество. Конечно, это предложение содержит только тавтологию, которая не расширяет наше знание. Оно содержит истину, которая является настолько очевидной, что кажется бессодержательной... Действительно, посредством одних определений нельзя доказать истину, которал без них не была бы доказуема" [4].

Это является, в свою очередь, обоснованием их применения в ходе рассуждений.

Если нами принято определение $A=\operatorname{def} B$, то в любом выражении языка $T[A]$, в которое входит определяемое, мы можем заменить его на определяющее и получить выражение $T[B / A]$. Это правило замены кажется настолько очевидным, что его широко используют люди, даже не знакомые с тем, что такое логика.

C формальной точки зрения, к явным определениям предъявляются два основных требования [5]. Во-первых, введение посредством определений в язык новых символов должно удовлетворять условию консервативности. То есть не приводить к тому, что ранее недоказуемые утверждения языка $L$ становятся 
доказуемыми в расширенном языке $L^{+}$. Во-вторых, мы должны иметь возможность сводить выражения языка $L^{+}$к выражениям языка $L$, заменяя определяемое на определяющее. Второе требование как раз и разрешает использовать правило замены в ходе рассуждений. В явной форме эти два требования были впервые сформулированы К. Айдукевичем и С. Лесневским.

Логика, которую мы собираемся построить ниже, теснейшим образом связана с комбинаторной логикой Шейнфинкеля-Карри и $\lambda$-исчислением А. Чёрча [6].

\section{2 Логика дефинициальной дедукции}

Чтобы приступить к анализу определений, мы должны фиксировать язык. Если мы возьмем стандартный язык первопорядкового исчисления предикатов, то на наши результаты могут повлиять те неявные предпосылки, которые в нем принимаются. Мы бы этого не хотели. Наша цель - исследовать определения независимо от возможной категориальной структуры языка и наполнения его конкретными дескриптивными терминами. Нам нужен в определенном смысле пустой язык, который впоследствии мог бы быть расширен в любом направлении. Основная идея заключается в том, чтобы рассмотреть язык логического субъекта, который еще не приступил к познанию окружающего его мира и потому даже никак не категоризовал его. Мы не первые сталкиваемся с подобной проблемой, и ее стандартным решением является бестиповый язык. Он не содержит ничего, кроме переменных, которые представляют всего лишь возможные объекты мысли.

\section{1 Исходные символы языка}

1. Var - множество переменных;

2. Const - множество констант, которое изначально пусто;

3. = def - символ для введения определений;

4. ), ( - скобки.

Выражения этого языка будем называть термами. Они являются просто комбинациями переменных и констант, структурированными посредством скобок. Изначально констант в языке 
нет, но мы допускаем, что они могут быть введены в него позже.

\section{2 Термы}

1. Если $x \in \operatorname{Var}$, то $x$ - терм;

2. Если $c \in$ Const, то $c$ - терм;

3. Если $X$ и $Y$ - термы, то $(X Y)$ - терм;

4. Ничто другое термом не является.

Терм вида $(X Y)$ не предполагает никакой подразумеваемой интерпретации. Это просто синтаксическая рядоположенность двух термов, которым могут соответствовать любые два объекта мысли. Взятие термов в скобки всего лишь на максимально абстрактном уровне отражает тот эмпирический факт, что выражения языка структурированы.

Поскольку при нашем определении терма он может содержать много скобок, будем для облегчения восприятия опускать лишние скобки, предполагая их ассоциацию влево при восстановлении. Например, терм $((((X) Y) Z) U)$ после опускания скобок может быть записан просто как $X Y Z U$, а терм $((X(Y Z)) U)$ примет вид $X(Y Z) U$.

В дальнейшем изложении будем использовать символ $\equiv$ для обозначения графического равенства термов как языковых выражений.

\section{3 Одновременная подстановка}

Посредством $T\left[Z_{1} / x_{1}, \ldots, Z_{n} / x_{n}\right]$ или $T\left[\overrightarrow{Z_{n} / x_{n}}\right]$ будем обозначать результат одновременной подстановки термов $Z_{1}, \ldots, Z_{n}$ в терм $T$ вместо всех вхождений переменных $x_{1}, \ldots, x_{n}$, определяемый следующим образом:

- $x_{i}\left[\overrightarrow{Z_{n} / x_{n}}\right] \equiv Z_{i}$, где $1 \leq i \leq n$

- $y\left[\overrightarrow{Z_{n} / x_{n}}\right] \equiv y$, где $y$ - атомарный терм, отличный от $x_{i}$, $1 \leq i \leq n$

- $(X Y)\left[\overrightarrow{Z_{n} / x_{n}}\right] \equiv\left(X\left[\overrightarrow{Z_{n} / x_{n}}\right]\right)\left(Y\left[\overrightarrow{Z_{n} / x_{n}}\right]\right)$. 
Кроме термов, объектный язык содержит конструкции, называемые определениями. Необходимо обратить внимание на то, что определения принадлежат не метаязыку, а именно объектному языку.

Константы, введенные определениями, и составленные лишь из них термы будем выделять жирным шрифтом.

Обозначим посредством $F V(T)$ множество всех переменных, входящих в терм $T$.

\section{4 Определения}

Если $T$ - терм, $F V(T) \subseteq\left\{x_{1}, \ldots, x_{n}\right\}, n \geq 0$ и $\mathbf{D}-$ константа, то $\mathbf{D} x_{1} \ldots x_{n}={ }_{\operatorname{def}} T$ - определение.

В случае, когда $n>0$, новый термин, представленный константой D, определяется вместе с контекстом его употребления. Такие определения называются контекстуалъными. Если $n=0$, то определение нового термина не зависит от контекста, т.е. является неконтекстуальным.

Каждое применение операции определения приводит к расширению констант языка и множества его термов. Чтобы отразить это в используемой нами символике, мы должны принять некоторые соглашения.

\section{5 Соглашения}

1. Пусть $\Delta-$ некоторое множество определений. Посредством Const $(\Delta)$ будем обозначать множество всех констант, введенных в язык определениями $\Delta$, т.е. $\operatorname{Const}(\Delta)=\{\mathbf{D}$ : $\left.\mathbf{D} x_{1} \ldots x_{n}={ }_{\text {def }} T \in \Delta\right\}$.

2. Посредством $L(\Delta)$ будем обозначать множество всех правильно построенных термов в языке с Const $=\operatorname{Const}(\Delta)$.

Операцию введения определений представим в виде правила.

\section{6 Правило введения определений}

Если имеется множество определений $\Delta$, терм $T$ и константа $\mathbf{D}$, удовлетворяющие ограничениям $T \in L(\Delta)$ и $\mathbf{D} \notin L(\Delta)$, то мы можем расширить множество $\Delta$ посредством нового определения $\mathbf{D} x_{1} \ldots x_{n}={ }_{\operatorname{def}} T$. 


\section{(DI) $\quad \Delta, T \in L(\Delta), \mathbf{D} \notin L(\Delta) \quad \Rightarrow \quad \Delta \cup\left\{\mathbf{D} x_{1} \ldots x_{n}==_{\text {def }} T\right\}$}

Мы должны учесть, что определения вводятся последовательно и поэтому в определенном смысле согласованы.

\section{7 Согласованное множество определений}

Множество определений $\Delta$ называется согласованным, е. и т. е. существует такая последовательность множеств $\left\langle\Delta_{0}, \ldots, \Delta_{n}\right\rangle$, что $n \geq 0, \Delta_{0}=\emptyset, \Delta_{n}=\Delta$, и для всех $0<i \leq \mathrm{n}$ множество $\Delta_{i}$ получено из $\Delta_{i-1}$ путем применения правила введения определений DI.

С формальной точки зрения, посредством определений логический субъект всего лишь запоминает, каким образом из переменных $x_{1}, \ldots, x_{n}$, входящих в левую часть определения, и, возможно, уже содержащихся в языке констант, может быть построен терм, находящийся в правой части определения. Новая константа языка, определяемый термин, репрезентирует структуру определяющего. После принятия определения этот новый абстрактный объект вводится в универсум рассуждения и становится самостоятельным объектом мысли логического субъекта, а соответствующая ему константа может участвовать в построении новых термов. В обыденной практике, определив, что значит разделить одно число на другое, мы начинаем говорить об операции деления, которой овладели, как о самостоятельном объекте. Необходимо особо отметить, что новый абстрактный объект существует независимо от какой-либо интерпретации языка, так как его значением является структура языкового терма, который мы построили и выбрали на роль дефиниенса.

\section{8 Правило замены}

Если $\mathbf{D} x_{1} \ldots x_{n}=_{d e f} T$ - определение, а $X\left\{\mathbf{D} Y_{1} \ldots Y_{n}\right\}$ - терм, с выделенным конкретным вхождением терма $\mathbf{D} Y_{1} \ldots Y_{n}$, то $X\left\{T\left[\overrightarrow{Y_{n} / x_{n}}\right]\right\}$ есть результат замены $\mathbf{D} Y_{1} \ldots Y_{n}$ согласно определению на $T\left[\overrightarrow{Y_{n} / x_{n}}\right]$.

$$
\text { (DE) } \mathbf{D} x_{1} \ldots x_{n}={ }_{\text {def }} T, X\left\{\mathbf{D} Y_{1} \ldots Y_{n}\right\} \quad \Rightarrow \quad X\left\{T\left[\overrightarrow{Y_{n} / x_{n}}\right]\right\}
$$

При кажущейся сложности формулировки правила замены оно не представляет ничего необычного. Вспомним, как мы вво- 
дим в язык логики высказываний определение связки эквиваленции

$$
p \leftrightarrow q={ }_{\text {def }}(p \supset q) \&(q \supset p) .
$$

Встретив формулу $A \vee(B \leftrightarrow C)$ и пожелав устранить эквиваленцию в соответствии с принятым определением, мы должны подставить в дефиниенс вместо пропозициональной переменной $p$ формулу $B$, подставить вместо переменной $q$ формулу $C$, т.е. выполнить операцию одновременной подстановки $((p \supset q) \&(q \supset$ $p))[B / p, C / q]$, получить в результате формулу $(B \supset C) \&(C \supset B)$ и после этого произвести замену $(B \leftrightarrow C)$ на $(B \supset C) \&(C \supset B)$. Результатом замены будет формула $A \vee((B \supset C) \&(C \supset B))$. Именно эта последовательность шагов и отражена в нашей формулировке правила замены.

\section{9 Дефинициальной дедукцией}

(выводом) терма $X$ из согласованного множества определений $\Delta$ и конечного множества термов $\Sigma \subseteq L(\Delta)$ называется непустая конечная последовательность $<X_{0}, \ldots, X_{n}>$ термов, где $X_{n}=X$ и каждый член которой либо является элементом $\Sigma$, либо получен по правилу DE из предшествующих термов последовательности и определений $\Delta$.

В дальнейшем изложении всякий раз, говоря о множестве определений, мы будем подразумевать, что оно является согласованным.

Запись $\Delta ; \Sigma>X$ будет означать, что существует вывод терма $\mathrm{X}$ из множества определений $\Delta$ и множества посылок $\Sigma$. В тех случаях, когда множество определений $\Delta$ фиксировано, и это не может вызвать недоразумений, мы будем использовать сокращенную запись $\Sigma>X$.

\section{3 Свойства логики дефинициальной дедукции}

ЛЕММА 1. Въвод $\Delta ; \Sigma>X$ имеет место, е. и т. е. для некоторого терма $Y \in \Sigma$ имеет место вывод $\Delta ;\{Y\}>X$.

Лемма доказывается простой индукцией по построению вывода. Базис индукции очевиден, а доказательство шага индукции следует из того, что посылка правила DE состоит из одного определения и одного терма. 
Благодаря этой лемме при доказательстве свойств логики дефинициальной дедукции нам достаточно рассматривать выводы лишь из одноэлементного множества термов $\Sigma$. В дальнейшем вместо $\Delta ;\{Y\}>X$ будем использовать запись $\Delta ; Y>X$.

ЛЕММА 2. Логика дефиниииальной дедукиии обладает свойством Чёрча-Россера. Если имеют место выводы $\Delta ; X>Y$ u $\Delta ; X>Z$, то существует такой терм $U$, что иметот место въводы $\Delta ; Y>U$ u $\Delta ; Z>U$.

Доказательство этой леммы выходит за рамки настоящей статьи.

Из свойства Чёрча-Россера следует, что в логике дефинициальной дедукции отношение выводимости обладает функциональными свойствами и поэтому может быть использовано для представления функций.

Следующая теорема говорит о том, что какие бы определения мы ни принимали, это никогда не сделает логику противоречивой в смысле тривиальности.

TEOPЕМА 3 (нетривиальности). Для любого множества определений $\Delta$ существуют такие термы $X$ и $Y$, что вывод $\Delta ; X>$ $Y$ не имеет места.

\section{Доказательство.}

Если множество определений $\Delta$ пусто, то нетривиальность следует из того, что все выводы имеют вид $\emptyset ; X>X$.

Если множество определений $\Delta$ непусто, то оно содержит хотя бы одно контекстуальное определение вида $\mathbf{D} x_{1} \ldots x_{n}={ }_{d e f} T$, где $n>0$. Очевидно, что вывод $\Delta ; \mathbf{D}>(\mathbf{D D})$ не имеет места, так как ни для одного определения из множества $\Delta$ правило $\mathrm{DE}$ к константе $\mathbf{D}$ не применимо, ибо ее контекст не заполнен. Q.E.D.

Определения должны удовлетворять условию консервативности. Доказательство этого свойства является содержанием следующей теоремы.

TEОРЕМА 4 (о консервативности определений). Если для множества определений $\Delta$ и термов $X, Y \in L(\Delta)$ не верно, ито имеет место въвод $\Delta ; X>Y$, то для любого нового определения $\boldsymbol{D} x_{1} \ldots x_{n}={ }_{\text {def }} T$ въвод $\Delta \cup\left\{\boldsymbol{D} x_{1} \ldots x_{n}={ }_{\text {def }} T\right\} ; X>Y$ также не будет иметь места. 


\section{Доказательство.}

Допустим, что для $X, Y \in L(\Delta)$ имеет место вывод $\Delta \cup$ $\left\{\mathbf{D} x_{1} \ldots x_{n}=_{\text {def }} T\right\} ; X>Y$. Покажем, что в этом случае также будет иметь место вывод $\Delta ; X>Y$. Доказательство проводим индукцией по построению вывода $\Delta \cup\left\{\mathbf{D} x_{1} \ldots x_{n}={ }_{d e f} T\right\} ; X>Y$.

Базис, $Y=X$

Так как $X \in L(\Delta)$, то имеет место вывод $\Delta ; X>X$.

Индукционный шаг. Достаточно рассмотреть случай, когда терм $Y$ имеет вид $Y\left\{U\left[\overrightarrow{Z_{k} / x_{k}}\right]\right\}$ и получен по правилу DE из некоторого предшествующего терма последовательности $Y\left\{\mathbf{W} Z_{1} \ldots Z_{k}\right\}$ и определения $\mathbf{W} x_{1} \ldots x_{k}==_{\text {def }} U \in \Delta \cup\left\{\mathbf{D} x_{1} \ldots\right.$ $\left.x_{n}={ }_{\text {def }} T\right\}$.

По индуктивному допущению имеет место вывод $\Delta ; X>$ $Y\left\{\mathbf{W} Z_{1} \ldots Z_{k}\right\}$, но в этом случае $Y\left\{\mathbf{W} Z_{1} \ldots Z_{k}\right\} \in L(\Delta)$ и, следовательно, $\mathbf{W} x_{1} \ldots x_{k}={ }_{\text {def }} U \in \Delta$. Отсюда получаем вывод $\Delta ; X>Y\left\{U\left[\overrightarrow{Z_{k} / x_{k}}\right]\right\}$.

Q.E.D.

Важнейшим свойством комбинаторной логики, $\lambda$-исчисления Чёрча и логики дефинициальной дедукции является наличие неподвижных точек для любого терма и существование эффективного алгоритма для их нахождения.

TEOPEMA 5 (о неподвижных точках). Для всякого множества определений $\Delta$ и всякого терма $F \in L(\Delta)$ существует такой терм $X \in L\left(\Delta \cup\left\{\boldsymbol{A} x y={ }_{\text {def }} y(x x y)\right\}\right)$, ито имеет место вывод $\Delta \cup\left\{\boldsymbol{A} x y==_{\text {def }} y(x x y)\right\} ; X>F X$.

\section{Доказательство.}

Пусть $F \in L(\Delta)$ - произвольный терм. В качестве искомого терма $X$ возьмем $\mathbf{A A} F$.

1. $\mathbf{A} \mathbf{A} F$

2. $y(x x y)[\mathbf{A} / x, F / y]-$ из 1 по $\mathrm{DE}$

3. $F(\mathbf{A} \mathbf{A} F)-$ из 2 по опр. 2.3

4. $F X-$ из 1,3

Q.E.D. 
СЛЕДСТВИЕ 6. Пусть $\Delta-$ некоторое множество определений, а $C$ - произвольный терм в языке $L(\Delta)$. Обозначим посредством $\Theta$ множество определений $\Delta \cup\left\{\boldsymbol{G} x_{0} x_{1} \ldots x_{n}={ }_{\text {def }} C\right.$, $\left.\boldsymbol{A} x y={ }_{\text {def }} y(x x y)\right\}$. Тогда существует такой терм $\boldsymbol{F} \in L(\Theta)$, что для всяких термов $T_{1}, \ldots, T_{n} \in L(\Theta)$ имеет место вывод $\Theta ; \boldsymbol{F} T_{1} \ldots T_{n}>C\left[\boldsymbol{F} / x_{0}, T_{1} / x_{1}, \ldots, T_{n} / x_{n}\right]$.

В качестве искомого терма $\mathbf{F}$ возьмем $\mathbf{A A G}$.

1. $\mathbf{A A G} T_{1} \ldots T_{n}$

2. $(y(x x y)[\mathbf{A} / x, \mathbf{G} / y]) T_{1} \ldots T_{n}-$ из 1 по $\mathrm{DE}$

3. $\mathbf{G}(\mathbf{A A G}) T_{1} \ldots T_{n}-$ из 2 по опр. 2.3

4. $C\left[(\mathbf{A A G}) / x_{0}, T_{1} / x_{1}, \ldots, T_{n} / x_{n}\right]-$ из 3 по $\mathrm{DE}$

5. $C\left[\mathbf{F} / x_{0}, T_{1} / x_{1}, \ldots, T_{n} / x_{n}\right]-$ из $1-4$

Для тех, кто специально не интересовался понятием неподвижной точки, необходимо пояснить, что это такое, и почему оно действительно представляет интерес.

Одной из стандартных задач арифметики является решение уравнений вида $f(x)=0$. Очевидно, что это уравнение имеет в точности те же корни, что и уравнение $f(x)=x-x$, которое, в свою очередь, может быть преобразовано к виду $f(x)+x=x$. Обозначим $f(x)+x$ посредством $g(x)$. Получаем, что задача решения исходного уравнения $f(x)=0$ равносильна решению уравнения $g(x)=x$, т.е. нахождению таких $x$, при которых значение функции $g(x)$ совпадает со значением ее аргумента. Отсюда и появился термин неподвижная точка. Ряд глубоких теорем математики формулируется в терминах существования неподвижных точек.

Теорема 5 и следствие 6 говорят о том, что каждый терм дефинициальной логики имеет неподвижную точку, и эту точку (терм) можно эффективно построить. Благодаря этому мы можем расширить нашу логику новым видом определений, которые позволяют вводить в язык новые константы посредством самореференции. В обычной логике таких определений стараются избегать, считая самореференцию порочным кругом и ошибкой. Ограниченная форма определений посредством саморефе- 
ренции используется в математике под названием рекурсивных определений.

\section{1 Определения через неподвижные точки}

Если $T-$ терм, $F V(T) \subseteq\left\{x_{0}, x_{1}, \ldots, x_{n}\right\}$, и $\mathbf{D}-$ константа, то $\mathbf{D} x_{1} \ldots x_{n}=_{d f p} T\left[\mathbf{D} / x_{0}\right]-$ определение через неподвижную точку.

\section{2 Правило введения определений через неподвижную точку}

Если имеется множество определений $\Delta$, терм $T$ и константа $\mathbf{D}$, удовлетворяющие ограничениям $T \in L(\Delta)$ и $\mathbf{D} \notin L(\Delta)$, то мы можем расширить множество $\Delta$ посредством нового определения $\mathbf{D} x_{1} \ldots x_{n}={ }_{d f p} T\left[\mathbf{D} / x_{0}\right]$.

$(\mathrm{DFI}) \Delta, T \in L(\Delta), \mathbf{D} \notin L(\Delta) \Rightarrow \Delta \cup\left\{\mathbf{D} x_{1} \ldots x_{n}=_{d f p} T\left[\mathbf{D} / x_{0}\right]\right\}$

Очевидно, что в том случае, когда переменная $\mathrm{x}_{0}$ не имеет вхождений в терм $\mathrm{T}$, определения через неподвижную точку совпадают с обычными определениями.

\section{3 Расширенное согласованное множество определений}

Множество определений $\Delta$ называется расширенным согласованным, е. и т. е. существует такая последовательность множеств $<\Delta_{0}, \ldots, \Delta_{n}>$, что $n \geq 0, \Delta_{0}=\emptyset, \Delta_{n}=\Delta$ и для всех $0<i \leq n$ множество $\Delta_{i}$ получено из $\Delta_{i-1}$ путем применения правила введения определений DI или DFI.

\section{4 Правило замены}

(Для определений через неподвижную точку). Если $\mathbf{D} x_{1} \ldots$ $x_{n}={ }_{d f p} T\left[\mathbf{D} / x_{0}\right]-$ определение, а $X\left\{\mathbf{D} Y_{1} \ldots Y_{n}\right\}-$ терм, с выделенным вхождением терма $\mathbf{D} Y_{1} \ldots Y_{n}$, то $X\left\{T\left[\mathbf{D} / x_{0}, Y_{1} / x_{1}, \ldots\right.\right.$, $\left.\left.Y_{n} / x_{n}\right]\right\}$ есть результат замены $\mathbf{D} Y_{1} \ldots Y_{n}$ согласно определению на $T\left[\mathbf{D} / x_{0}, Y_{1} / x_{1}, \ldots, Y_{n} / x_{n}\right]$

$$
\begin{gathered}
(\mathrm{DFE}) \quad \mathbf{D} x_{1} \ldots x_{n}={ }_{d f p} T\left[\mathbf{D} / x_{0}\right], X\left\{\mathbf{D} Y_{1} \ldots Y_{n}\right\} \Rightarrow \\
\\
X\left\{T\left[\mathbf{D} / x_{0}, Y_{1} / x_{1}, \ldots, Y_{n} / x_{n}\right]\right\} .
\end{gathered}
$$

Нам остается показать, что определения через неподвижную точку не могут привести ни к чему плохому. 
TEOPЕМА 7 (о консервативности определений через неподвижную точку). Если для множества определений $\Delta$ и термов $X, Y \in L(\Delta)$ не верно, что имеет место вывод $\Delta ; X>Y$, то для любого нового определения $\boldsymbol{D} x_{1} \ldots x_{n}=_{d f p} T\left[\boldsymbol{D} / x_{0}\right]$ вывод $\Delta \cup\left\{\boldsymbol{D} x_{1} \ldots x_{n}=_{d f p} T\left[\boldsymbol{D} / x_{0}\right]\right\} ; X>Y$ также не будет иметь места.

\section{Доказательство.}

Допустим, что имеет место вывод $\Delta \cup\left\{\mathbf{D} x_{1} \ldots x_{n}={ }_{d f p} T\left[\mathbf{D} / x_{0}\right]\right\}$; $X>Y$. Покажем, что в этом случае также будет иметь место вывод $\Delta ; X>Y$.

Обозначим посредством $\Theta$ множество определений $\Delta \cup$ $\left\{\mathbf{A} x y={ }_{\text {def }} y(x x y), \mathbf{G} x_{0} x_{1} \ldots x_{n}={ }_{\text {def }} T\right\}$.

Во всех термах вывода $\Delta \cup\left\{\mathbf{D} x_{1} \ldots x_{n}={ }_{d f p} T\left[\mathbf{D} / x_{0}\right]\right\} ; X>Y$ заменим константу $\mathbf{D}$ на терм $\mathbf{A A G}$. Покажем, что результатом такой замены является вывод $\Theta ; X>Y$.

Доказательство проводим индукцией по построению вывода $\Delta \cup\left\{\mathbf{D} x_{1} \ldots x_{n}=_{d f p} T\left[\mathbf{D} / x_{0}\right]\right\} ; X>Y$.

a) $Y=X$.

Так как $X \in L(\Delta)$, то $\Theta ; X>X$.

b) В выводе $\Delta \cup\left\{\mathbf{D} x_{1} \ldots x_{n}=_{d f p} T\left[\mathbf{D} / x_{0}\right]\right\} ; X>Y$ терм $Y$ имеет вид $Y\left\{N\left[\overrightarrow{Z_{k} / x_{k}}\right]\right\}$ и получен по правилу DE из терма $Y\left\{\mathbf{S} Z_{1} \ldots Z_{k}\right\}$ и определения $\mathbf{S} x_{1} \ldots x_{k}=\operatorname{def} N \in \Delta$.

По индуктивному допущению, имеет место вывод

$$
\Theta ; X>U\left\{\mathbf{S} V_{1} \ldots V_{k}\right\}
$$

где $U, V_{1}, \ldots, V_{k}$ есть результат замены в термах $Y, Z_{1}, \ldots, Z_{k}$ константы $\mathbf{D}$ на терм AAG. Из терма $U\left\{\mathbf{S} V_{1} \ldots V_{k}\right\}$ применением правила DE получаем терм $U\left\{N\left[\overrightarrow{V_{k} / x_{k}}\right]\right\}$ и соответственно вывод $\Theta ; X>U\left\{N\left[\overrightarrow{V_{k} / x_{k}}\right]\right\}$.

c) В выводе $\Delta \cup\left\{\mathbf{D} x_{1} \ldots x_{n}=_{d f p} T\left[\mathbf{D} / x_{0}\right]\right\} ; X>Y$ терм $Y$ имеет вид $Y\left\{N\left[\mathbf{S} / x_{0}, Z_{1} / x_{1}, \ldots, Z_{n} / x_{k}\right]\right\}$ и получен по правилу DFE из терма $Y\left\{\mathbf{S} Z_{1} \ldots Z_{k}\right\}$ и определения $\mathbf{S} x_{1} \ldots x_{k}={ }_{d f p} N\left[\mathbf{S} / x_{0}\right]$.

По индуктивному допущению, имеет место вывод

$$
\Theta ; X>U\left\{\mathbf{S} V_{1} \ldots V_{k}\right\},
$$


где $U, V_{1}, \ldots, V_{k}$ есть результат замены в термах $Y, Z_{1}, \ldots, Z_{k}$ константы $\mathbf{D}$ на терм $\mathbf{A A G}$. Из терма $U\left\{\mathbf{S} V_{1} \ldots V_{k}\right\}$ применением правила DFE получаем терм $U\left\{N\left[\mathbf{S} / x_{0}, V_{1} / x_{1}, \ldots, V_{k} / x_{k}\right]\right\}$ и соответственно вывод $\Theta ; X>U\left\{N\left[\mathbf{S} / x_{0}, V_{1} / x_{1}, \ldots, V_{k} / x_{k}\right]\right\}$.

d) В выводе $\Delta \cup\left\{\mathbf{D} x_{1} \ldots x_{n}={ }_{d f p} T\left[\mathbf{D} / x_{0}\right]\right\} ; X>Y$ терм $Y$ имеет вид $Y\left\{T\left[\mathbf{D} / x_{0}, Z_{1} / x_{1}, \ldots, Z_{n} / x_{n}\right]\right\}$ и получен по правилу $\mathrm{DFE}$ из терма $Y\left\{\mathbf{D} Z_{1} \ldots Z_{n}\right\}$ и определения $\mathbf{D} x_{1} \ldots x_{n}={ }_{d f p} T\left[\mathbf{D} / x_{0}\right]$.

По индуктивному допущению, имеет место вывод

$$
\Theta ; X>U\left\{\mathbf{A A G} V_{1} \ldots V_{n}\right\}
$$

где $U, V_{1}, \ldots, V_{n}$ есть результат замены в термах $Y, Z_{1}, \ldots, Z_{n}$ константы D на терм A AG. Продолжим этот вывод.

1. $U\left\{\mathbf{A A G} V_{1} \ldots V_{n}\right\}-$ заключительный терм предшествующего вывода

2. $U\left\{(y(x x y)[\mathbf{A} / x, \mathbf{G} / y]) V_{1} \ldots V_{n}\right\}-$ из 1 по $\mathrm{DE}$

3. $U\left\{\mathbf{G}(\mathbf{A A G}) V_{1} \ldots V_{n}\right\}$ - из 2 по опр. 2.3

4. $U\left\{T\left[\mathbf{A A G} / x_{0}, V_{1} / x_{1}, \ldots, V_{n} / x_{n}\right]\right\}$ - из 3 по $\mathrm{DE}$

В результате мы получили вывод $\Theta ; X>U\left\{T\left[\mathbf{A A G} / x_{0}\right.\right.$, $\left.\left.V_{1} / x_{1}, \ldots, V_{n} / x_{n}\right]\right\}$.

Все случаи рассмотрены. Так как в выводе $\Theta ; X>Y$ по условию теоремы $X, Y \in L(\Delta)$, то мы можем применить к нему теорему 4 и получить искомый вывод $\Delta ; \Sigma>Y$ Q.E.D.

Из следствия 6 и теоремы 7 следует, что определения через неподвижную точку не расширяют дедуктивных возможностей дефинициальной логики, так как всегда могут быть заменены обычными определениями в смысле 2.4. Вместе с теоремой 4 это означает, что определения через неподвижную точку являются полноправными определениями и при корректном их использовании ни к чему плохому привести не могут.

Стандартной задачей арифметики является нахождение решения системы уравнений:

$$
\begin{aligned}
& f_{1}\left(x_{1}, \ldots, x_{n}\right)=0 \\
& \ldots \ldots \ldots \\
& f_{n}\left(x_{1}, \ldots, x_{n}\right)=0
\end{aligned}
$$


Эту задачу можно свести к задаче о нахождении множественных неподвижных точек для системы уравнений:

$$
\begin{aligned}
& f_{1}\left(x_{1}, \ldots, x_{n}\right)+x_{1}=x_{1} \\
& \ldots \ldots \ldots \ldots \\
& f_{n}\left(x_{1}, \ldots, x_{n}\right)+x_{n}=x_{n}
\end{aligned}
$$

Следующая теорема является усилением теоремы 5 .

TEOPEMA 8 (Теорема о множественных неподвижных точках). Для всякого множества определений $\Delta$ u термов $F_{1}, \ldots$, $F_{n} \in L(\Delta)$ существует такое множество определений $\Theta$ u терми $X_{1}, \ldots, X_{n} \in L(\Theta)$, что $\Delta \subseteq \Theta$ и имеют место виво$\partial ъ \Delta ; X_{1}>\left(F_{1} X_{1} \ldots X_{n}\right), \ldots, \Delta ; X_{n}>\left(F_{n} X_{1} \ldots X_{n}\right)$.

\section{Доказательство.}

В качестве $\Theta$ возьмем множество $\Delta$, к которому последовательно добавлены следующие определения:

$$
\begin{aligned}
& \mathbf{P}_{1} x_{1} \ldots x_{n}=d_{\text {def }} x_{1} \\
& \ldots \ldots \ldots \ldots \\
& \mathbf{P}_{n} x_{1} \ldots x_{n}={ }_{\text {def }} x_{n} \\
& \mathbf{R} x=_{d f p} x\left(F_{1}\left(\mathbf{R P}_{1}\right) \ldots\left(\mathbf{R P}_{n}\right)\right) \ldots\left(F_{n}\left(\mathbf{R P}_{1}\right) \ldots\left(\mathbf{R} \mathbf{P}_{n}\right)\right)
\end{aligned}
$$

В качестве терма $X_{i}$ возьмем $\mathbf{R P}_{i}$.

1. $\mathbf{R P}_{i}$

2. $x\left(F_{1}\left(\mathbf{R P}_{1}\right) \ldots\left(\mathbf{R} \mathbf{P}_{n}\right)\right) \ldots\left(F_{n}\left(\mathbf{R P}_{1}\right) \ldots\left(\mathbf{R} \mathbf{P}_{n}\right)\right)\left[\mathbf{P}_{i} / x\right]$ - из 1 по DFE

3. $\mathbf{P}_{i}\left(F_{1}\left(\mathbf{R P}_{1}\right) \ldots\left(\mathbf{R} \mathbf{P}_{n}\right)\right) \ldots\left(F_{n}\left(\mathbf{R P}_{1}\right) \ldots\left(\mathbf{R} \mathbf{P}_{n}\right)\right)$ - из 2 по опр. 2.3

4. $x_{i}\left[\left(F_{1}\left(\mathbf{R P}_{1}\right) \ldots\left(\mathbf{R} \mathbf{P}_{n}\right)\right) / x_{1}, \ldots,\left(F_{n}\left(\mathbf{R P}_{1}\right) \ldots\left(\mathbf{R} \mathbf{P}_{n}\right)\right) / x_{n}\right]$ - из 3 по $\mathrm{DE}$

5. $\left.F_{i}\left(\mathbf{R P}_{1}\right) \ldots\left(\mathbf{R P}_{n}\right)\right)$ - из 4 по опр. 2.3

$$
\text { Q.E.D. }
$$

В настоящей статье мы не будем приводить точных формулировок и доказательств, но лишь заметим, что из теоремы 8 по аналогии с теоремой 5 вытекает возможность принятия множественных определений через неподвижные точки. В арифметике 
аналогом этого является одновременное определение нескольких функций посредством взаимной рекурсии.

ТЕОРЕМА 9. Логика дефинищиальной дедукции эквивалентна исчислению редукиий комбинаторной логики ШейнфинкеляКарри [6].

Подробное доказательство этой теоремы выходит за рамки настоящей статьи. Дадим лишь его набросок.

В логике дефинициальной дедукции мы можем определить два основных комбинатора исчисления редукций комбинаторной логики:

$\mathbf{K} x y={ }_{\operatorname{def}} x$

$\mathbf{S} x y z={ }_{\text {def }} x z(y z)$

После этого остается лишь показать допустимость в дефинициальной логике правил вывода исчисления редукций комбинаторной логики.

Доказательство в обратную сторону использует тот факт, что правило введения определений DI является аналогом свойства комбинаторной полноты исчисления редукций. Остается доказать допустимость в исчислении редукций правила DE. Это легко сделать индукцией по глубине вхождения терма $\mathrm{D} Y_{1} \ldots Y_{n}$ в терм $X\left\{\mathbf{D} Y_{1} \ldots Y_{n}\right\}$.

\section{4 Приложения логики дефинициальной дедукции}

Удивительным свойством логики дефинициальной дедукции является то, что многие известные математические объекты и конструкции могут быть в ней просто определены, а не постулированы, как в обычной логике предикатов. Определения этих объектов и их существование не зависят от понятия модели, так как имеют чисто языковую природу.

\section{1 Соглашения}

1. Будем говорить, что терм, к которому не применимы правила DE и DFE, находится в нормалъной форме.

2. Если из терма $X$ выводим находящийся в нормальной форме терм $Y$, то будем говорить, что терм $X$ uмеет нормальную форму. 
Из свойства Чёрча-Россера для дефинициальной логики следует, что если терм имеет нормальную форму, то она единственна. Этот факт удобно использовать для представления функций.

Допустим, мы хотим определить в нашем языке некоторую функцию $\mathbf{f}: A^{n} \rightarrow B$, где $A$ и $B$ - некоторые множества. Для этого каждому элементу $a \in A$ и каждому $b \in B$ необходимо сопоставить термы $\ulcorner a\urcorner$ и $\ulcorner b\urcorner$, которые будут их представлять. Должно быть соблюдено условие: если $a_{1} \in A, a_{2} \in A$ и $a_{1} \neq$ $a_{2}$, то $\left\ulcorner a_{1}\right\urcorner$ и $\left\ulcorner a_{2}\right\urcorner$ имеют разные нормальные формы, т.е. не существует такого терма $t$, что $\left\ulcorner a_{1}\right\urcorner>t$ и $\left\ulcorner a_{2}\right\urcorner>t$. Аналогично для $b_{1} \in B, b_{2} \in B$.

\section{2 Определимость функций}

Функция $\mathbf{f}: A^{n} \rightarrow B$ определима в логике дефинициальной дедукции, если существует такой замкнутый терм $\mathbf{F}$, что для всяких $a_{1}, \ldots, a_{n} \in A$ имеет место вывод $\mathbf{F}\left\ulcorner a_{1}\right\urcorner \ldots\left\ulcorner a_{n}\right\urcorner>\left\ulcorner f\left(a_{1}, \ldots\right.\right.$, $\left.\left.a_{n}\right)\right\urcorner$, где $\left\ulcorner f\left(a_{1}, \ldots, a_{n}\right)\right\urcorner$ представляет элемент $\mathbf{f}\left(a_{1}, \ldots, a_{n}\right) \in B$. В этом случае будем говорить, что терм $\mathbf{F}$ определяет функцию $\mathrm{f}$.

\section{3 Определение истинностнозначных булевых функций}

Определим две константы, которые будут представлять в нашем языке Истину и Ложъъ.

1. $(\mathbf{T} x y)=\operatorname{def} x$

2. $(\mathbf{F} x y)=\operatorname{def} y$

Поскольку термы $\mathbf{T}$ и $\mathbf{F}$ находятся в нормальной форме и отличны друг от друга, то необходимые условия соблюдены. Определим теперь дополнительные константы, представляющие основные булевы функции.

1. $(\operatorname{Not} x)=_{\text {def }}(x \mathbf{F} \mathbf{T})$

2. $($ And $x y)={ }_{\text {def }}(x y \mathbf{F})$

3. $($ Or $x y)=_{\text {def }}(x \mathbf{T} y)$ 
4. $(\operatorname{Imp} x y)=_{\operatorname{def}}(x y \mathbf{T})$

Легко проверить, что они действительно представляют булевы функции. Проверим это на примере констант Not и And. Для остальных функций проверка аналогична.

$$
\begin{aligned}
& (\operatorname{Not} \mathbf{T})>\left(\begin{array}{lll}
\mathbf{T} & \mathbf{F} & \mathbf{T}
\end{array}\right)>\mathbf{F} \\
& (\operatorname{Not} \mathbf{F})>\left(\begin{array}{lll}
\mathbf{F} & \mathbf{F} & \mathbf{T}
\end{array}\right)>\mathbf{T}
\end{aligned}
$$

Этот пример демонстрирует общую схему, как определяются функции в языке дефинициальной логики. Подобным образом можно показать, что в ней определимы не только булевы, но и все (!) функции рекурсивной арифметики.

Поскольку исходный язык пуст, т. е. не содержит никаких дескриптивных терминов с заранее заданной интерпретацией, арифметика рекурсивных функций развивается за счет его внутренних ресурсов и потому совместима с любыми будущими расширениями. Это явление может быть названо лингвистическим априоризмом.

Мы не можем остановиться лишь на констатации данного факта, а должны дать ему какое-то рациональное объяснение. Повидимому, оно заключается в том, что многие структуры языка, не только искусственно нами построенного, но и естественных языков, являются древовидными. Эти структуры обнаруживаются при анализе слов, предложений, текстов. Их богатые выразительные возможности для кодирования информации и представления вычислений хорошо известны в математике. Поэтому совершенно не удивительно, что обнаружив их в языке, мы открыли в себе способность с помощью операций введения определений (кодирования) и замены согласно определениям (декодирования) представлять разнообразные математические объекты и конструкции. А поскольку наше знание мы всегда формулируем и закрепляем в языке, то оно вынуждено подчиняться этим математическим структурам. Не в этом ли и заключается ответ на вопрос о том, почему математика столь успешно приложима к внешнему миру? 


\section{Литература}

[1] Brown J.R. Philosophy of Mathematics. Routledge, 1999. P. 74.

[2] Бочаров В.А., Маркин В.И. Основы логики. Учебник. М.: Космополис, 1994. C. 201.

[3] Whitehead A.N., Russel B. Principia Mathematica. Vol. 1. Cambridge, 1910. P. 11.

[4] Фреге Г. Логика в математике. Избранные работы. М.: Дом интеллектуальной книги, 1997. С. 101.

[5] Gupta A. Definitions // The Stanford Encyclopedia of Philosophy / Edward N. Zalta (ed.), URL $=<$ http://plato.stanford.edu/entries $/$ definitions $/>$.

[6] Барендрегт X. Ламбда-исчисление. Его синтаксис и семантика. М.: Мир, 1985. 